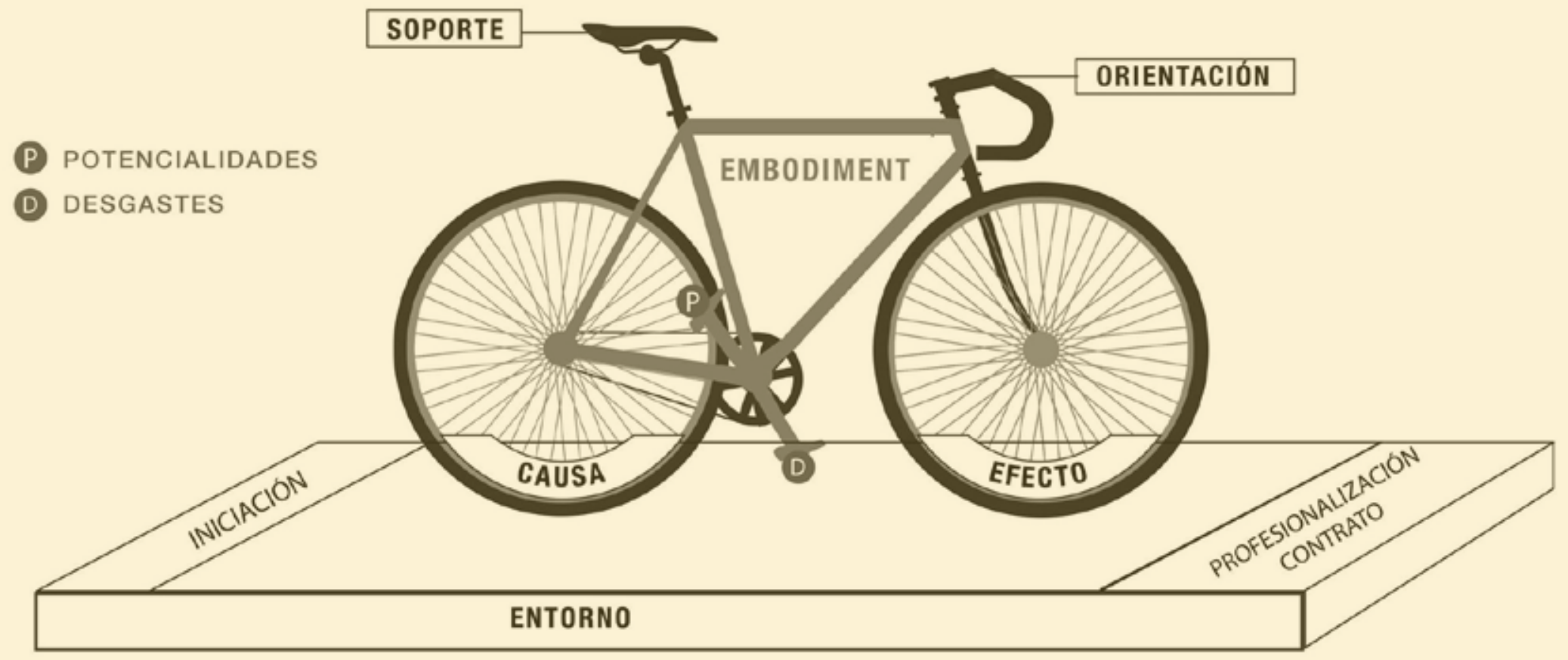

HISTORICIDAD DEL PROCESO FORMATIVO

Sistema Categorial. Elaborado por Robayo, A y Anzola, J. (2014). (Anzola-Moreno \& Robayo-Torres 2016). 


\section{Relatos biográficos y experiencias encarnadas en ciclistas profesionales colombianos: tránsitos y po- sibilidades ${ }^{1}$}

Biographical stories and embodiment experiences in colombian professional cyclists: transits and possibilities // Narrativa pessoal e experiências encarnadas em ciclista profissional colombiana: trânsitos e possibilidades

Javier Anzola Moreno²

Universidad Nacional de Colombia

jaanzolam@unal.edu.co

Aydee Robayo Torres ${ }^{3}$

Universidad Nacional de Colombia

alrobayot@unal.edu.co

Revista Corpo-grafías: Estudios críticos de y desde los cuerpos / Volumen 7 - Número 7 / Enero - diciembre 2020 / ISSN impreso 2390-0288, ISSN digital 2590-9398 / Bogotá, D.C., Colombia / 256-266

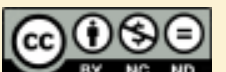

Fecha de recepción: 29 de enero de 2019

Fecha de aceptación: 22 de mayo de 2019

DOI: https://doi.org/ 10.14483/25909398.15523

Cómo citar este artículo: Anzola Moreno, J., Robayo Torres, A. (2020, enero-diciembre). Relatos biográficos y experiencias encarnadas en ciclistas profesionales colombianos: tránsitos y posibilidades. Revista Corpo-grafías: Estudios críticos de y desde los cuerpos, 7(7), pp. 256-266 / ISSN 2390-0288.

\footnotetext{
$1 \quad$ Articulo de reflexión Investigación: El presente artículo deriva de la investigación realizada por Javier Andrés Anzola Moreno, bajo la orientación de Aydee Luisa Robayo Torres en el marco del Máster en Fisioterapia del deporte y la actividad física de la Universidad Nacional de Colombia.

$2 \quad \mathrm{Ft}$, MsC en Fisioterapia del Deporte y la Actividad Física. Profesor experto. Departamento del Movimiento Corporal Humano - Universidad Nacional de Colombia.

2 Ft, MsC en Desarrollo Educativo y Social, PhD. Profesora asociada. Departamento del Movimiento Corporal Humano, Facultad de Medicina, Universidad Nacional de Colombia.
} 


\section{Resumen}

En los paradigmas del deporte, el cambio en la noción del cuerpo máquina a la corporeidad del deportista, evidencia la necesidad de abordar al sujeto deportista desde la dimensión subjetiva e intersubjetiva, valorando significados y sentidos de su práctica deportiva a partir del cuerpo vivido en relatos biográficos, que encarnan los desgastes y potencialidades de la categoría de cuerpo-ciclista. El presente artículo muestra los tránsitos y posibilidades de las narrativas de la corporeidad en el ciclismo, comunicando al mundo la necesidad de ser reconocidos como humanos, no como máquinas. Al comprender esta realidad, el diálogo del cuerpo con el entorno a través del dispositivo subjetivado (la bicicleta), permite reconocer las funciones cognitiva, práctica e identitaria de estas narraciones, tanto en el ciclista que narra, como en quien investiga. Es habitar la palabra, en este caso: pedaleada, es decir, incorporada y significada.

\section{Palabras clave}

Biografía; Ciclismo; Narrativa Personal

\section{Abstract}

In the paradigms of sport, the change in the notion of the machine body to the athlete's embodiment, evidences the need to approach the athlete subject from the subjective and intersubjective dimension, valuing meanings and senses of his sports practice from the body lived in stories biographical, which embody the wear and potential of the body-cyclist category. This article shows the transits and possibilities of embodiment narratives in cycling, communicating to the world the need to be recognized as human, not as machines. By understanding this reality, the body's dialogue with the environment through the subjectivized device (the bicycle), allows recognizing the cognitive, practical and identity functions of these narratives, both in the cy- clist who narrates, and in the researcher. It is to inhabit the word, in this case: pedaled, that is, incorporated and meaning.

\section{Keywords}

Biography; Bicycling; Personal Narrative

\section{Resumo}

Nos paradigmas do esporte, a mudança da noção de corpo da máquina para a corporeidade do atleta, evidencia a necessidade de abordar o sujeito atleta a partir da dimensão subjetiva e intersubjetiva, valorizando significados e sentidos de sua prática esportiva a partir do corpo vivido nas histórias biográfico, que incorporam o desgaste e o potencial da categoria ciclista-corpo. Este artigo mostra os trânsitos e as possibilidades das narrativas no ciclismo, comunicando ao mundo a necessidade de serem reconhecidos como humanos, não como máquinas. Ao entender essa realidade, o diálogo do corpo com o meio ambiente, por meio do dispositivo subjetivado (a bicicleta), permite reconhecer as funções cognitivas, práticas e identitárias dessas narrativas, tanto no ciclista que narra quanto no pesquisador. É habitar a palavra, neste caso: pedalada, isto é, incorporada e significado.

\section{Palavras-chaves}

\section{Biografia; Ciclismo; Narrativa Pessoal}

\section{Enunciación}

Las vidas significadas a partir del cuerpo vivido y la narración de quienes experimentan el deporte, desde la formación hasta la alta competencia, no han sido llevadas a un escenario de conocimiento desde la corporeidad narrada, valorada y atravesada por la subjetividad 
y la intersubjetividad. Más allá de la representación de un cuerpo objetivo, extensión del laboratorio donde se ha desarrollado mayoritariamente el conocimiento de las ciencias aplicadas al deporte, por una perspectiva dominante en el positivismo y en la materialidad del cuerpo, desconociendo su lugar social e implicación en la vida de los sujetos; una valoración socio-cultural que desde el ciclismo colombiano se manifiesta y se usa como un medio pacificador o interlocutor de valores, pero donde paradójicamente, el deportista como sujeto encarnado, es atacado y violentado ante el logro deportivo no obtenido. El presente artículo muestra el desarrollo metodológico de tipo histórico-hermenéutico que se abarcó en el estudio sobre narrativas de la corporeidad en ciclistas colombianos durante su proceso formativo (Anzola-Moreno \& Robayo-Torres, 2016) realizado durante el 2015 como parte de la investigación en la Maestría en fisioterapia del deporte y la actividad física, y los ecos que en estos años posteriores han surgido de la categoría de cuerpo ciclista a partir de dichas narraciones, su valor metodológico y las manifestaciones en voces de ciclistas profesionales que ante los medios y la presión social, aluden a las categorías centrales de discusión en este estudio realizado, vigente y que, en paralelo al desarrollo del ciclismo colombiano actual, luego de lograr un ciclista colombiano el título de campeón del Tour de Francia, cobran mayor sentido metodológico, conceptual y pragmático ante las contradicciones de comercialización, hegemonías y condiciones de vida de los ciclistas en formación.

\section{Alistamiento}

El alistamiento, ese ritual que comienza días anteriores a la competencia y donde el ciclista presta atención a los detalles de su preparación, de su bicicleta y su indumentaria. Conoce y anticipa el recorrido, hace de la rutina una fuente de seguridad y proyecta la estrategia para afrontar la competencia. Así constituyó para Javier (autor, ciclista, entrenador y fisioterapeuta), la preparación de este viaje metodológico para ser interlocutor de quienes veía pedalear en Europa, sus contemporáneos, algunos de ellos amigos desde niños; y traer sus narraciones desde el cuerpo vivido a un ámbito investigativo que significara sus vidas como ciclistas profesionales "World Tour" y dar lugar a la corporeidad del ciclista como uno de los tránsitos en la concepción del ciclismo y en general del deporte, puesto que pone en tensión los paradigmas con los cuales se ha abordado el deporte de alta competencia como lo expresan (Carbinatto, Moreira, Nunomura, Tsukamoto, \& Nista-Piccolo, 2011) al decir "que en la comunidad de las ciencias del deporte ha sido históricamente reflejada la idea positivista de la ciencia, sobre todo por el paradigma mecanicista/reduccionista que ha guiado el camino de profesionales que tratan a los atletas y el de todo el conocimiento producido. La disposición para simplificar la naturaleza y el mundo por partes ha fragmentado y reducido investigaciones en compartimientos, más no en el intercambio de información entre los científicos". Una invitación a repensar la forma en que vemos a los ciclistas, a nosotros mismos (como profesionales en el ámbito del deporte y la salud) y al cuerpo colectivo disciplinar que rige hegemónicamente el deporte de alto rendimiento.

La investigación surge de las reflexiones propias de Javier (he aquí autor) de su propio proceso de formación en el ciclismo y de ver sus contemporáneos estar en Europa, llevando consigo esas ilusiones y sueños, pero que tomaron un sentido académico al ser entrenador y fisioterapeuta para alistarse a un mundo de potencias investigativas y llegar a ser interlocutor de quienes han vivido (disfrutado-sufrido) el ciclismo profesional a nivel mundial.

El propósito de comprender el sentido de la experiencia encarnada sobre la bicicleta durante un proceso formativo, que denota proyecto de vida en el ciclismo profesional, y la significación del cuerpo que se forma en el mundo de los pedales, del vínculo afectivo con la bici- 
cleta y el poder heroico de ciclistas colombianos de los años 70's y 80's que de nuevo encarna la montaña en la llamada" Nueva era de oro" del ciclismo colombiano actual, fue el norte de la metodología y la manifestación de lo que otros ciclistas "World Tour", durante y luego de unos años de realizado el estudio, reclamaran a los medios de comunicación y a la sociedad: una valoración social de su humanidad en disputa por los resultados competitivos, un llamado al cambio de paradigmas en el deporte con relación al cuerpo.

\section{El encuentro y las voces}

Los estudios sociales del cuerpo en el deporte, con los recursos teóricos de la fenomenología, han sido relativamente escasos (Hockey \& Allen-Collinson, 2007). Igualmente, desde las narrativas de deportistas colombianos a partir de las historias de vida, refiriendo únicamente un estudio en deporte paralímpico (Bajaña, 2010).

En relación con el ciclismo, no se hallaron estudios relacionados con la corporeidad y el proceso formativo, siendo relevante investigar sobre esta temática dado el auge del ciclismo actual en Colombia y sus implicaciones sociológicas en la formación deportiva.

Como marco referencial del estudio, se tomó la noción biográfica de las historias de vida para la metodología, situando al cuerpo vivido y la corporeidad desde el embodiment como categoría central en las potencialidades y los desgastes. La recolección de información se hizo a través de entrevista abierta a cuatro ciclistas profesionales, tres hombres y una mujer, vinculados en ese momento a equipos reconocidos por la Unión Ciclística Internacional- UCl y nacidos entre 1987 y 1990. Con los cuales acordábamos el encuentro de manera virtual, dada su estancia en otras ciudades de Colombia y en su mayoría, en Europa. Siguiendo la metodología del estudio referido por (Pascual, 2009) en deportistas retirados en España, la elección de los criterios para seleccionar a los ciclistas entrevistados buscaba una representatividad de tipo "estructural», en el sentido de que estos autores buscaban aproximarse a deportistas retirados para comprender la realidad social desde los significados que estos asignaban a sus experiencias, partiendo del supuesto de que sus discursos individuales reproducen discursos sociales y de que la peculiaridad de estos discursos individuales depende precisamente de su ubicación en la estructura social (Pascual, 2009). De ahí que el tipo de entrevista tenía un guion a partir de la categoría central (corporeidad desde los degastes y las potencialidades) pero que facilitaba, dada la experiencia del entrevistador en el ciclismo, la exploración de las dimensiones objetiva, subjetiva e intersubjetiva al interactuar con los ciclistas.

La entrevista fue un encuentro, no solo por la posibilidad de interactuar, sino porque significó un reflejo de experiencias entre los ciclistas y el entrevistador, aludiendo a la memoria de situaciones compartidas o paralelas que daban lugar a una indagación de los relatos biográficos desde hechos concretos o ya percibidos por el entrevistador, para ir a profundidad en las narraciones, aludiendo a la vivencia corporal como una percepción auténtica del movimiento atravesada por el contexto y en relación dialógica con el entorno, con el mundo, dando cuenta de la vida misma, sin separar los hechos cotidianos de los deportivos y donde el cuerpo es el nodo relacional.

Si el mundo y la vida se dan por el cuerpo, esto implica asumir el cuerpo como reflexivo, racional, sensible, trascendente y no caracterizándolo como algo menos o como obstáculo para el conocimiento, citando a Loland, (2006). La corporeidad es una actitud única comprensible a través de una reconexión de conocimiento al tratar de conocer el cuerpo-problema y el cuerpo-misterio (Carbinatto et al., 2011). Para la sociedad, estos ciclistas profesionales representan los valores heroicos producto de los logros competitivos, pero el misterio radica en poder conocer el proceso de preparación, y más allá, aquel proceso de formación para "llegar a 
ser" (ciclista "World Tour"). Un cuerpo-problema en el sentido que se desconoce su constitución y requiere ser estudiado, noción que invita a las preguntas sobre ese cuerpo, sobre el que pedalea "en" y "por el mundo". Esta intención debe superar la dualidad de las ciencias, y sería posible a través de una concepción ontológica del ser en el que su presencia en el mundo definiría su identidad. El cuerpo "no es un objeto, ni una idea, es la expresión única de lo humano en movimiento". Separar el sujeto y el objeto es reducir el complejo cuerpo humano (Carbinatto et al., 2011).

Al tratar de comprender la fenomenología del cuerpo en el deporte, tomamos principalmente la obra de Merleau-Ponty, de especial relevancia dada su principal preocupación con la conciencia encarnada, citada por Meier, (1988), el cuerpo como "ser-en-el-mundo". La misión Merleau- Ponty era revelar: "por debajo del objeto y el conocimiento individual del cuerpo que otro conocimiento que tenemos de él en virtud de su estar siempre con nosotros mismos y el hecho de que nosotros somos nuestro cuerpo ", citando a Merleau- Ponty, (1962). Desde este punto de vista, el cuerpo no es tanto un instrumento ni un objeto, sino el sujeto de la percepción, socialmente y de hecho, subculturalmente mediada. Para nosotros, que conocemos el mundo a través del cuerpo, del mismo modo que el cuerpo produce el mundo para nosotros, de modo que: "El cuerpo vivido no es sólo una cosa en el mundo, sino una manera en la que el mundo llegaría a ser" citado por Leder (1992). Además, como Luijpen (1966) explica, nuestras manos no son viejas manos, sino que más bien son "mi propio agarre de las cosas"; nuestros pies no son solo partes del cuerpo en algún debate de anatomía, sino que participan en la 'mi caminata'. Por otra parte, para Merleau-Ponty esta percepción está inextricablemente vinculada con el movimiento, y todo movimiento corporal se acompaña de la intencionalidad que se encuentra en el núcleo de la percepción (Hockey \& Allen-Collinson, 2007). Dado el interés de Mer-
leau-Ponty en la conciencia humana como conciencia encarnada, en la percepción, la intencionalidad y las formas en que experimentamos el tiempo/espacio vivido, su obra es particularmente apropiada, y un pequeño pero creciente corpus se basa en la fenomenología existencial al examinar el deporte y actividades física (Allen-Collinson, 2009) y que tomamos como referencia conceptual para el abordaje metodológico en este estudio, puesto que las narraciones de estos ciclistas aludían a su corporeidad durante el proceso formativo y permitían comprenderla desde la "materialidad de la existencia humana, potencialidades y desgastes; una materialidad orgánica, anatómica y morfológica sobre la que recaen los procesos salud-enfermedad", donde se hacen visibles las prácticas influenciadas por la cultura que se transforman en el tiempo (Torres M, 2004) y pueden servirse de la perspectiva de la encarnación desarrollada en las ciencias sociales para ampliar su comprensión de la experiencia encarnada y situada, así como de las prácticas performativas para la generación de acontecimientos que potencien la actividad sensible y la transformación individual y colectiva (García Gutiérrez, 2011).

Las voces hechas narraciones al expresar vivencias corporales significativas y significadas, aluden a lo biográfico y su importancia metodológica para comprender las realidades en que los sujetos son en el mundo. La comprensión del mundo por parte de cada sujeto, en definitiva, está en función de los relatos a los que tiene acceso y con los que interactúa, construyendo su propia interpretación a partir de las prácticas que desarrolla (Rivas \& Pastor, 2009). El proceso de categorización en el contexto del análisis de las prácticas narrativas, el investigador establece categorías, se plantea la construcción de tipologías. Es importante tener en cuenta que existen categorías que la sociedad establece y les asigna a los actores. Las categorías tienen un papel de lentes sociales, "sirven para mirar con mayor nitidez cómo funciona el "orden social", develan su realidad con sus 


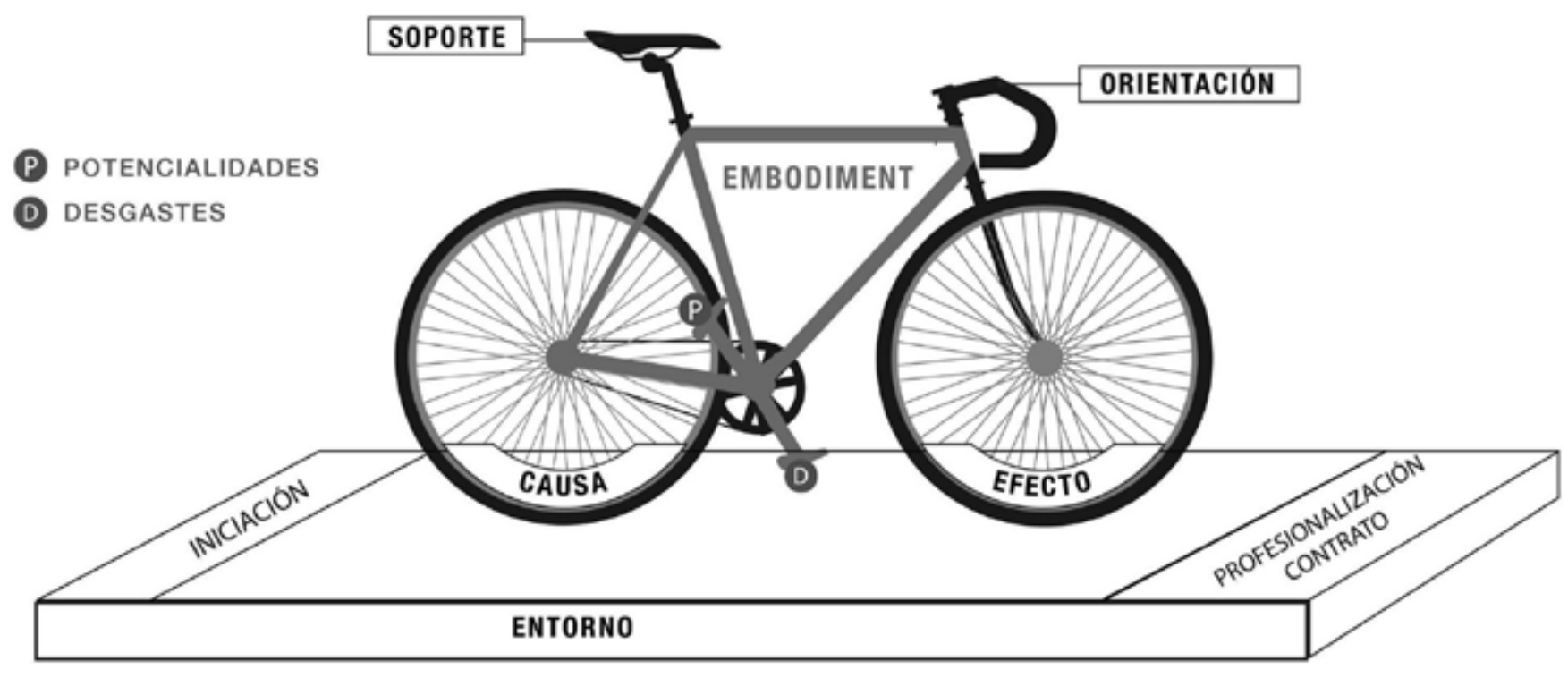

HISTORICIDAD DEL PROCESO FORMATIVO

Imagen 1. Sistema Categorial. Elaborado por Robayo, A y Anzola, J. (2014). (Anzola-Moreno \& Robayo-Torres 2016).

más y sus menos, con sus filos cortantes e hirientes, con sus desagarraduras, con sus pactos sociales Ochoa, Á J (1997), (citado por (Baja, 2015)). Sin embargo, más allá de estos lentes es generar una identidad narrativa, como lo expresa (Sparkes, 2004), al decir que las narrativas proporcionan una estructura para nuestro propio sentido de individualidad e identidad. Es decir, cuando contamos historias sobre nuestras vidas a nosotros mismos y a los demás, creamos una identidad narrativa (Sparkes, 2004), un proceso de reflexión y resignificación del cuerpo, fuera de estereotipos típicos del cuerpo en el deporte como aquel disciplinado, dominante y comunicativo, y la forma en que operan estos tipos de cuerpos en diferentes contextos del ciclismo élite, haciendo alusión al análisis de la autobiografía de Lance Amstrong, especialmente desde el cuerpo y la enfer- medad, realizada por (Sparkes, 2004). En la complejidad del deporte, el análisis del cuerpo y la corporeidad a partir de las narraciones biográficas toman relevancia desde la epistemología de las historias de vida, entendidas como registros culturales que permiten interpretar y comprender la realidad, favorecen la construcción de sentidos vitales, permiten escuchar voces y significados del pasado y del presente. Ciertamente tienen unas metodologías que más que universalizar resultados, buscan la construcción de sentido, significar la vida de los seres humanos participantes (Baja, 2015) como lo pretendió la investigación que antecede a este artículo y que se reafirma en el tiempo con las voces y narraciones de los propios ciclistas, donde sus cuerpos entran en escenarios de disputa institucional, política y social. 


\section{Tránsito y manifestaciones}

Como parte del tránsito de la investigación, la identificación de las categorías centrales y emergentes, y su interpretación generó como resultados las siguientes nociones de relevancia y han dado lugar a discusión donde se ha presentado el estudio. El cuerpo ciclista, categoría constituyente del significado y el sentido de la vida de los ciclistas profesionales entrevistados, emerge de las narraciones y la interpretación de un ciclista investigador para la comprensión de estas vidas, para invitar a la reflexión y el cambio en el pensamiento sociológico en el abordaje de procesos de formación deportiva hacia la alta competencia de ciclistas. Acompañado necesariamente, de preguntas al conceptualizar esta categoría como la interacción biológica-subjetiva con el entorno a través de un cuerpo (humano-bicicleta) gestor de identidad, de una forma única y auténtica de ver, ser y llegar a ser/hacer en el mundo, con disfrute, pasión y sufrimiento.

La corporeidad narrada desde las potencialidades y desgastes, en relación con las categorías del proceso formativo en el ciclismo, da significado a la vida de los ciclistas y sentido a sus vivencias, como parte de la identidad del cuerpo-ciclista y la trama constitutiva del cuerpo como territorio en la episteme de lo cotidiano, a pesar del paradigma predominante del cuerpo-máquina en el deporte. Estos modos de sentir y valorar son la expresión de las transformaciones corporales, incluidos los cambios físicos y cognitivos propios del ciclismo, a los que sería imposible darles significado sin la emotividad de descubrirse como individuo ciclista, al tanto de las restricciones y potencialidades de su cuerpo. La búsqueda de sí mismo en la juventud es una preocupación constante orientada a la construcción de una autoimagen satisfactoria. Ese proceso de construcción propicia maneras propias de enfrentar la vida, presenta señales de singularidad individual reflejadas en las prácticas elegidas con relativa autonomía, citando a Torres y Munevar (2004), pues parece que el ciclista en su juventud se apropia de una identidad $-\mathrm{y}$ el sentido personal que se le asigna- derivada de su cuerpo, a la que puede acceder a través del deporte, visto éste como forma de enfrentar la vida en la historicidad del proceso formativo y las categorías como el entorno al cual está expuesto, donde la montaña es determinante, el soporte y la orientación del proceso formativo puesto en familia y el entrenador principalmente, las causas y los efectos de una iniciación en el ciclismo hasta la profesionalización mediada por un contrato, categorías expuestas en el sistema categorial simbólico de la figura $\mathrm{N}^{\circ} 1$, formulado por el trabajo investigativo y donde se configuran las relaciones categoriales emergentes (Anzola-Moreno \& Robayo-Torres, 2016).

La subjetividad de las experiencias encarnadas-narradas, integra la vivencia del placer y el dolor, es decir, las potencialidades y los desgastes, ubicados simbólicamente en el sistema categorial en los pedales, pues su relación es ambivalente en cada experiencia del ciclista. Ambivalencia que, para el caso del cuerpo ciclista, representa la capacidad de sufrimiento como una única y auténtica potencialidad del ciclista que se forma, coexistiendo la potencialidad y el desgaste durante el proceso formativo, éste que se cree, finaliza con la firma de un contrato profesional, pero que se desmiente en el experimentar las potencialidades y los desgastes aun siendo profesional, a medida que se afrontan los retos de las competencias en términos de los rivales, la distancia, la topografía y las condiciones climáticas, o de querer "conquistar" las tres grandes vueltas del ciclismo mundial, formulando así, una extensión de la historicidad de lo que se piensa que es el proceso formativo.

En esta ambivalencia, hay un escenario especial de descubrimiento del sí mismo, que aludiendo a una experiencia de desgaste como es la lesión deportiva (derivada de un accidente principalmente), se convierte en una potencialidad. Este escenario emerge del encuentro 
con fisioterapia y el tránsito por la rehabilitación, la cual surge como un proceso de reconfiguración corporal, pasando de la fragmentación de la corporeidad (el cuerpo en partes y la pérdida de contacto con la bicicleta y el entorno de entrenamiento, con su carga subjetiva) a la noción del cuerpo reconocido como un "todo" para mejorar el desempeño en competencia (Anzola-Moreno \& Robayo-Torres, 2016) y tomar conciencia de sí mismo, como acto pedagógico implícito.

Se ha explorado, a partir de construcciones narrativas realizadas por atletas jóvenes, la percepción de sí mismos a lo largo de diferentes edades. Tal acercamiento es importante por varias razones. En primer lugar, abre una puerta significativa dentro de la literatura respecto a la comprensión de sí mismos a través del tiempo, mientras envejecen. Para estos individuos, el paso del tiempo y sus conexiones con los inevitables cambios de su cuerpo pueden dar lugar a una pérdida en sus ejecuciones atléticas. En segundo lugar, a partir de las narraciones que evidencian el curso de la vida, el atleta joven realiza una fluida y dinámica interacción entre sí mismo, su cuerpo y su contexto social. De esta manera se empieza a comprender cómo sus experiencias en el presente, pueden formar las opiniones del futuro, y cómo sus opiniones sobre el futuro pueden influenciar sus experiencias en el presente, como expresan Phoenix, C. y Sparkes, A. (2007) (Baja, 2015).

En este sentido, las manifestaciones de voces de ciclistas colombianos venían desde el año 2013 reclamando su humanidad, su corporeidad en el presente y dando opiniones para el futuro. Enfrentando a los medios de comunicación, y a través de ellos, a una sociedad sensacionalista y llena de heroísmo, de conquistas y guerras, imaginarios transferidos a los ciclistas en la competencia. Pues desde allí, amedrentaron en el 2013, 2016 y 2017 a ciclistas colombianos "World Tour" por las altas expectativas y/o el no poder ganar el Giro de Italia, el Tour de Francia, la Vuelta a España o los Juegos Olím- picos de Rio de Janeiro en 2016. Sus voces en respuesta a esto, dieron lugar a una narración en el tiempo durante estos años donde reclamaban diciendo:

"los ciclistas no somos máquinas"(Colprensa, 2013).

"Decirles que vamos a hacer oro, es muy difícil porque no todo es estadísticas y números, y somos humanos, no máquinas y al final lo que vamos a hacer es dejar la piel en la carretera. Vamos por buen camino" (RCNradio, 2016).

"No es para decepcionarse. No somos máquinas, no siempre sucede lo que uno quiere"; "No sabemos si se podría haber hecho mejor, pero estamos en el podio, que es lo importante. Hemos trabajado mucho, pero no siempre hay premio. Seguimos creciendo, sumando experiencias. Me quedó con eso"(Diario AS, 2017). "No estoy decepcionado; no somos máquinas para ganar todo"(Cabrera, 2017).

"No lo sabremos hasta que pase. Somos humanos. Lo que puede pasar no se puede medir. No somos máquinas. Hay que ir día a día"; "No me obsesiona eso. Hay que ir poco a poco. Si todo va bien no será el único ni el último Tour que haré. Vendrán muchos más. Si viene el triunfo de etapa, bienvenido sea y si no viene lo intentaremos otras veces en el futuro"( La FM radio, 2017)

Al momento, estas expresiones han dado relevancia a lo expresado por los ciclistas entrevistados, porque es en el pedalear que se han tejido estas narraciones, en el encuentro entre ellos y lo que expresan, con su autenticidad y donde al año 2019, en la etapa número 21 del Tour de Francia y siendo 28 de julio de 2019, se posaban cuatro ciclistas colombianos al mundo televisivo en frente del pelotón mundial de ciclistas para celebrar, que por primera vez en la historia un colombiano de 22 años de edad, ganaba la prueba épica del ciclismo en el mundo: el Tour de Francia. Presente y futuro de cuerpos ciclistas que se forman, que experimentan la potencialidad y el desgaste, pero más aún, la potencialidad del sufrimiento y disfrutar de éste, de darle sentido y significado en la analogía del pedalear por la vida, en relación íntima con la bicicleta, donde 
se construyen identidades y donde también nacen preguntas como ¿Cuáles serán las narraciones de este punto en adelante?, una pregunta para seguir en esta línea de episteme metodológica para el abordaje del deportista como eje de transformación social desde las dimensiones objetiva, subjetiva e intersubjetiva, y las demandas disciplinares y relacionales para cuidar de nuestros ciclistas como sujetos, como seres humanos que denotan un modo de ver, ser y llegar a ser/hacer en el mundo (Anzola-Moreno \& Robayo-Torres, 2016).

\section{Posibilidades de la metodología narrativa}

Proponer este camino investigativo nos obliga a develar nuestra certeza de comprender la narrativa como un modo de construir sentido por medio de la descripción y análisis de los datos biográficos (Castañeda, Clavijo Gloria María.; Gallo, 2007), es en estas producciones discursivas que entramos en el campo del lenguaje libre del entrevistado (Bustamante Zamudio, 2012) , ese lenguaje de la corporeidad hecha carne y experiencia, que trasciende al "tecnolecto" con el cual se pretende legitimar con grados de verosimilitud la investigación que rescata el aporte biográfico.

Reconstruir ese sí mismo, esa subjetividad entendida no sólo como "punto de vista de la persona, de lo que se es y las tensiones a las que está sometido", o bien como "ese "pensar en voz alta"(Candioti, 2006), la apuesta concreta en esta investigación es por una subjetividad que es "trama de percepciones, aspiraciones, memorias, saberes y sentimientos que nos impulsa y nos da orientación, incluso como un modo de transitar hacia otras formas de organización (Boisier, 2001) o de desorganización de la experiencia; una subjetividad que ameritaba ser develada (Robayo, 2015)
Una subjetividad que de acuerdo con el profesor Torres, cumpliría varias funciones, a saber:

- Cognitiva: como esquema referencial, posibilita la construcción de realidad.

- Práctica: pues desde ella los sujetos orientan y elaboran su experiencia

- Identitaria: pues aporta los materiales desde los cuales los individuos y colectivos definen su identidad personal y su sentido de pertenencia sociales (Torres Carrillo, 2007).

Creímos y lo seguimos creyendo, que es desde a opción de los actos vitales de la conversación, la narración, los relatos de la palabra compartida, con los deportistas, que se permitía que las preguntas lo y la habite, y que es desde allí que su saber fenomenológico como deportistas, que puede comprender la subjetividad que se transforma en cada práctica, en cada lesión, en los contratos profesionales prometidos, en los afectos y las afectaciones que el ciclismo anida, reproduce, habita, y nunca mejor dicho: In-corpora. Se pone de manifiesto, por tanto, una visión conflictiva y crítica de la realidad, que rompe con la visión del mundo organizada del funcionalismo. La complejidad es la característica más relevante de la realidad de la vida cotidiana y las narraciones biográficas se convierten en el modo de poder entrar en su mundo de significados (Rivas \& Pastor, 2009), y de los sentidos biográficos de las experiencias encarnadas, en el tránsito de la significación de la vida deportiva y la invitación a reconocer la realidad del ciclista, fuera de la pantalla de transmisión de las etapas, como si se adentrara el relato en lo cotidiano y sensible, para encontrarse en la palabra y la presencia, de la palabra pedaleada. 


\section{Referencias}

Allen-Collinson, J. (2009). Sporting embodiment: Sports studies and the (Continuing) promise of phenomenology. Qualitative Research in Sport and Exercise, 1(3), pp. 279-296. https://doi. org/10.1080/19398440903192340

Anzola-Moreno, J., \& Robayo-Torres, A. L. (2016). Narrativas de la corporeidad en ciclistas colombianos durante su proceso formativo. Revista de La Facultad de Medicina, 64(3Sup), p. 113. https://doi. org/10.15446/revfacmed.v64n3sup.51319

Baja, R. D. (2015). HISTORIAS DE VIDA EN EDUCACIÓN FÍSICA Y DEPORTE Un emocionante camino investigativo por descubrir. (November).

Bajaña, R. D. (2010). Life history of a Colombian paralympic athlete. Educación Fisica y ..., pp. 95-101. Retrieved from http://www.cabdirect.org/abstracts/20113058343.html

Boisier, S. (2001). 1245948918.Desarrollo_Local_De_que_estamos_ hablando_2_.

Bustamante Zamudio, G. (2012). Investigación y subjetividad: ino quieres quedar excluido?, no te metas a investigar. (U. P. Nacional, Ed.). Retrieved from http://cursomagistral.blogspot.com/2013/02/ universidad-pedagogica-nacional.html

Cabrera, A. (2017). Nairo Quitana: Ahora voy a ir al Tour a tratar de ganarlo... es mi obligación. Retrieved from http://www.radiosantafe.com/2017/05/28/nairo-quitana-ahora-voy-a-ir-al-tour-a-tratar-deganarlo-es-mi-obligacion/\#more-403264

Candioti, M. E. (2006). Prácticas lingüísticas, configuración de sentidos y subjetividad. Revista Tópicos, 14(1666-485X). Retrieved from https://www.redalyc.org/pdf/145/14518444003.pdf

Carbinatto, M. V., Moreira, W. W., Nunomura, M., Tsukamoto, M. H. C., \& Nista-Piccolo, V. L. (2011). Paradigms shift in sport sciences: Body's focus. World Academy of Science, Engineering and Technology, 80(8), pp. 1090-1094.

Castañeda, Clavijo Gloria María.; Gallo, C. L. E. (2007). Narrativa Corporal : una experiencia vivida a través de la danza. pp. 1-20. Retrieved from www.viref.udea.edu.co/contenido/pdf/133-narrativa.pdf

Colprensa. (2013). Rigoberto Urán: "Los ciclistas no somos máquinas." Retrieved from https://www.eluniversal.com.co/deportes/rigoberto-uran-los-ciclistas-no-somos-maquinas-133926-EQEU222240
Diario AS, C. (2017). Nairo: "No somos máquinas, no siempre se puede ganar." Retrieved from https://colombia.as.com/colombia/2017/05/28/masdeporte/1495989655_986416.html

García Gutiérrez, C. E. (2011). Hermenéutica de la educación corporal. Medellín: Funámbulos Editores.

Hockey, J., \& Allen-Collinson, J. (2007). Hockey, J and Allen-Collinson, J (2007) Grasping the Phenomenology of Sporting Bodies, International Review for the Sociology of Sport, 42(2), pp. 115-131. https://doi.org/0.1177/1012690207084747

La FM radio. (2017). Esteban Chaves, ante lo duró que le ha resultado el Tour: "no somos máquinas." Retrieved from https://www. lafm.com.co/deportes/esteban-chaves-ante-lo-duro-le-ha-resultado-tour-no-somos-maquinas

Pascual, A. S. (2009). Estrategias y prácticas cualitativas de investigación social. In Política y Sociedad 46. https://doi.org/10.5209/ POSO.22963

RCNradio. (2016). "Vamos por buen camino", dice Esteban Chaves sobre los olímpicos. Retrieved from https://www.rcnradio.com/deportes/buen-camino-dice-esteban-chaves-los-olimpicos

Rivas, J. I., \& Pastor, D. H. (2009). Voz y educación La narrativa como enfoque de interpretación de la realidad. Barcelona: Editorial Octaedro. Barcelona.

Robayo, A. L. (2015). Subjetividades docentes en la universidad pública colombiana. Comunidades de práctica a propósito de sus narraciones // Teaching Subjectivities in Colombian Public Universities . Communities of Practice According to their own Narratives // Subjetividades. Revista Colombiana de Educación, 68, pp. 229-263.

Sparkes, A. C. (2004). Bodies, narratives, selves, and autobiography: The example of Lance Armstrong. Journal of Sport and Social Issues, 28(4), 397-428. https://doi.org/10.1177/0193723504269907

Torres Carrillo, A. (2007). Identidad y política de la acción colectiva Bogotá: Universidad Pedagógica Nacional.

Torres M, M. DI. (2004). Representaciones corporales Bogotá: Editorial Divulgación académica y cultural; Universidad Nacional de Colombia. 


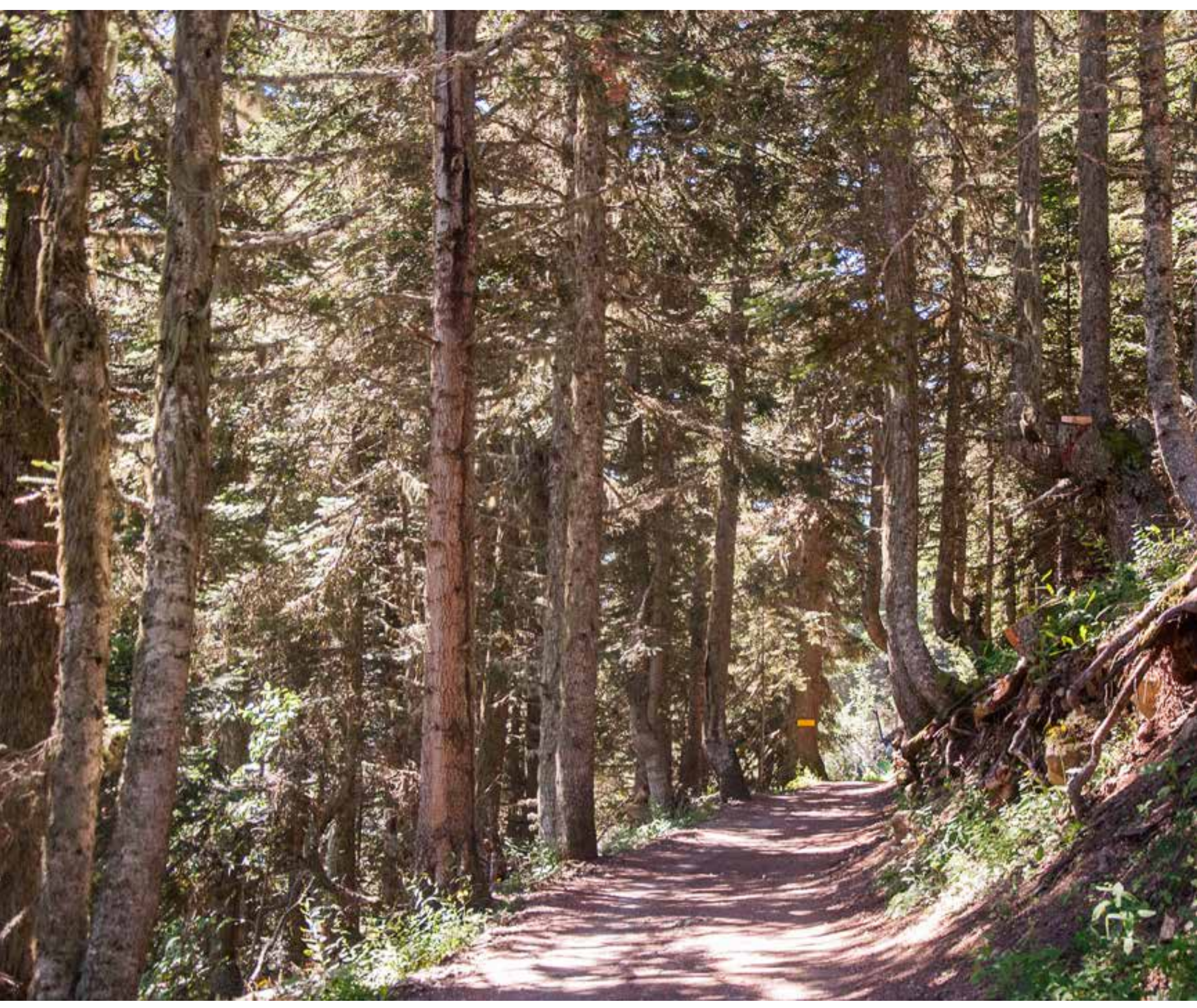

Parque Nacional los Pirineos (Francia, 2019). Fotografía: Karen Nathalia Díaz. 